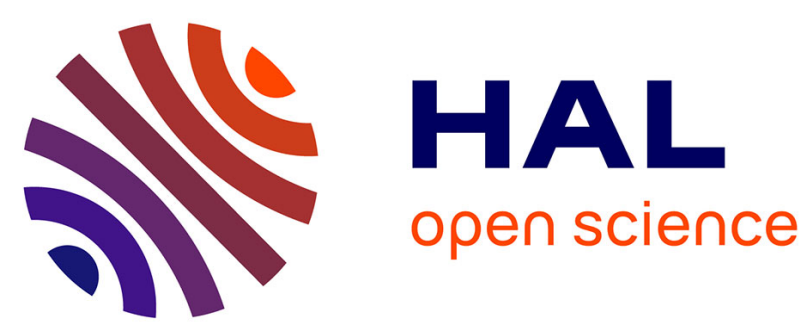

\title{
Stability analysis of some classes of input-affine nonlinear systems with aperiodic sampled-data control
}

Hassan Omran, Laurentiu Hetel, Mihaly Petreczky, Jean-Pierre Richard, Francoise Lamnabhi-Lagarrigue

\section{- To cite this version:}

Hassan Omran, Laurentiu Hetel, Mihaly Petreczky, Jean-Pierre Richard, Francoise LamnabhiLagarrigue. Stability analysis of some classes of input-affine nonlinear systems with aperiodic sampleddata control. Automatica, 2016, 70, pp.266-274. 10.1016/j.automatica.2016.02.013 . hal-01343851

\section{HAL Id: hal-01343851 \\ https://hal.science/hal-01343851}

Submitted on 10 Jul 2016

HAL is a multi-disciplinary open access archive for the deposit and dissemination of scientific research documents, whether they are published or not. The documents may come from teaching and research institutions in France or abroad, or from public or private research centers.
L'archive ouverte pluridisciplinaire HAL, est destinée au dépôt et à la diffusion de documents scientifiques de niveau recherche, publiés ou non, émanant des établissements d'enseignement et de recherche français ou étrangers, des laboratoires publics ou privés. 


\title{
Stability analysis of some classes of input-affine nonlinear systems with aperiodic sampled-data control ${ }^{\star}$
}

\author{
Hassan Omran ${ }^{\text {a }}$, Laurentiu Hetel ${ }^{\mathrm{b}}$, \\ Mihaly Petreczky ${ }^{\mathrm{b}}$ Jean-Pierre Richard ${ }^{\mathrm{b}, \mathrm{c}}$ Francoise Lamnabhi-Lagarrigue ${ }^{\mathrm{d}}$ \\ ${ }^{a}$ Ecole des Mines de Douai, Department of Computer Science and Automatic Control, F-59500 Douai, France \\ ${ }^{\mathrm{b}}$ Centre de Recherche en Informatique, Signal et Automatique de Lille (CRIStAL) UMR CNRS 9189, Ecole Centrale de Lille, \\ 59651 Villeneuve d'Ascq, France \\ ${ }^{\mathrm{c}}$ INRIA, Project NON-A, Lille, France \\ ${ }^{\mathrm{d}}$ L2S (CNRS UMR 8506), European Embedded Control Institute (EECI), SUPELEC, 3 rue Joliot Curie, 91192 \\ Gif-sur-Yvette, France
}

\begin{abstract}
In this paper we investigate the stability analysis of nonlinear sampled-data systems, which are affine in the input. We assume that a stabilizing controller is designed using the emulation technique. We intend to provide sufficient stability conditions for the resulting sampled-data system. This allows to find an estimate of the upper bound on the asynchronous sampling intervals, for which stability is ensured. The main idea of the paper is to address the stability problem in a new framework inspired by the dissipativity theory. Furthermore, the result is shown to be constructive. Numerically tractable criteria are derived using linear matrix inequality for polytopic systems and using sum of squares technique for the class of polynomial systems.
\end{abstract}

Key words: Nonlinear systems, sampled-data systems, aperiodic sampling, stability analysis, emulation approach.

\section{Introduction}

Recent years have witnessed an increasing number of works on stability analysis of nonlinear sampled-data systems. This challenging problem is of great interest since in applications practical controllers are often implemented digitally.

\footnotetext{
ऋ The research leading to these results has received funding from the European Community's 7th Framework Program (FP7/2007-2013) HYCON2 Network of Excellence (grant agreement No 257462), Region Nord-Pas de Calais, France (ESTIREZ project) and ANR project ROCC-SYS (agreement ANR-14-CE27-0008). The material in this paper was partially presented at the 13th European Control Conference (ECC 13), July 17-19, Zurich, Switzerland.

Email addresses: hassan.omran@mines-douai.fr (Hassan Omran), laurentiu.hetel@ec-lille.fr (Laurentiu Hetel), mihaly.petreczky@ec-lille.fr (Mihaly Petreczky), jean-pierre.richard@ec-lille.fr (Jean-Pierre Richard), francoise.lamnabhi-lagarrigue@lss.supelec.fr (Francoise Lamnabhi-Lagarrigue).
}

When implementing a controller digitally, the emulation approach is often considered [34]. In this approach, a continuous-time controller is designed, next it is implemented using a sample-and-hold device. However, the digital implementation must preserve the stability of the continuous-time system. Intuitively, the sampling interval must be sufficiently small to ensure the stability $[20,6]$. Still, in practice it is difficult to maintain a constant sampling period during real-time control and the variations of the sampling interval may have a destabilizing effect $[10,13]$, even for small sampling intervals. A quantitative estimation of the so-called Maximum Sampling Interval MSI that ensures stability (under timevarying sampling intervals), is very important from the practical point of view. Several works in the literature target this problem (see for example $[34,23,24,28]$ ).

The case of linear sampled-data systems has been extensively studied. For the input delay approach, see $[14,12,42,29]$ where stability conditions are derived based on Lyapunov-Krasovskii functionals [43]. The works in [31] and [15] use tools from robust control theory. A polytopic approximation of the discrete-time 
model is used in $[19,9]$ to handle the sampling effect based on Lyapunov-Razumikhin functions. In the hybrid systems modeling approach [17], the sampled-data system is represented as an impulsive system [11] and stability is investigated using Lyapunov functions with discontinuities at the impulse times $[5,33]$. The previous works provide constructive methods to estimate the MSI, such as Linear Matrix Inequalities (LMIs) based criteria.

The problem is more challenging in the nonlinear case $[32,26]$. We cite as follows some recent works. In [34], the authors specialized the results on generic Networked Control Systems (NCSs) for the particular case of sampled-data systems; stability conditions are presented based on the hybrid systems theory. In [1], asymptotic stability of NCSs is studied using the same hybrid systems formulation; the Lyapunov functions are constructed with a sum of squares (SOS) techniques. The input delay approach is explored in [23] for the nonlinear case, where Razumikhin functions together with theory of vector Lyapunov functions have been used. The work in [28] also considers the input delay approach and it investigates the robustness of nonlinear systems, with respect to both sampling and delay. The approach is inspired by the Lyapunov-Krasovskii functional method.

Here, we investigate a new research direction for nonlinear affine systems. The considered approach is inspired by the notion of exponential dissipativity [18]. This notion was initiated by Willems [44]. Since its introduction, it has been attracting an increasing attention. Dissipativity can be used to study stability, passivity, robustness and it is useful in a large variety of analysis and design problems. It was motivated by passivity properties of electrical circuits and it can be seen as a generalized notion of abstract energy for dynamical systems. Recently, local asymptotic stability of bilinear sampleddata systems controlled by a linear state feedback has been considered in [36], using the analysis of contractive invariant sets and dissipativity theory. The obtained results are promising, but the extension for generic nonlinear systems is not trivial.

The purpose of this work is to extend our previous result in [36], concerning the analysis of bilinear sampled-data systems, to the case of input-affine nonlinear sampleddata systems. Dissipativity based conditions are used to estimate the MSI. The robustness with respect to variations of the sampling intervals is considered. The results are shown to be applicable for local and global analysis. Additionally, in order to show the effectiveness of the results, we study the particular cases of polytopic systems and polynomial systems. We apply the result to a benchmark example from the literature to show the usefulness of the proposed stability conditions.

The remainder of the paper is organized as follows: the

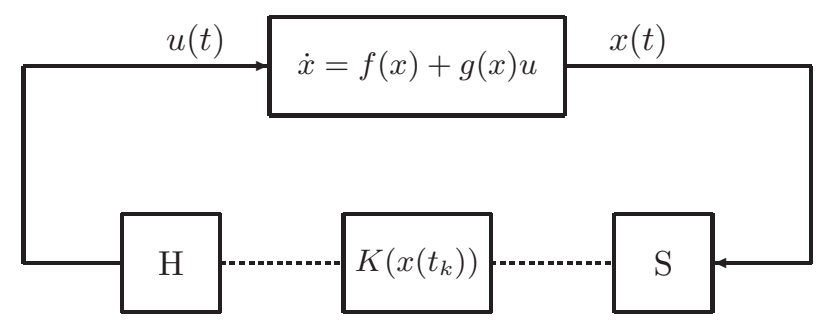

Fig. 1. Sampled-data feedback control of an affine nonlinear system.

problem under study is introduced in Section 2; in Section 3 , the system is represented by an equivalent model which is useful for our analysis; the main result is given in Section 4; case studies are presented in Section 5, where the main result is applied to the cases of polytopic and polynomial systems; finally, illustrative examples are presented in Section 6.

Notation: $\mathbb{R}$ is the set of real numbers and $\mathbb{R}^{+}$is the set of positive real numbers. $\mathbb{R}^{n}$ is the $n$-dimensional Euclidean space. The space of functions $f:[a, b) \rightarrow \mathbb{R}^{n}$ which are quadratically integrable over the interval $[a, b)$ is $L_{2}^{n}[a, b)$. The set of real matrices of dimension $n \times m$ is denoted by $\mathbb{R}^{n \times m}$. The transpose of a matrix $M$ is denoted by $M^{T}$. For $P \in \mathbb{R}^{n \times n}, P>0$ (resp. $P \geq 0$ ) means that it is a positive definite (resp. positive semidefinite) matrix. The identity matrix is $I$, the zero matrix is 0 , both with appropriate dimensions. For a given $M^{T}=M \geq 0$, the weighted inner product is denoted by $\langle x, y\rangle_{M}=x^{T} M y$, and the corresponding norm by $\|x\|_{M}=\sqrt{\langle x, x\rangle}_{M}$. The Euclidean norm is denoted by $|x|$. The convex hull is denoted by conv $\{\cdot\}$. The notation $p(\chi) \in \mathbb{R}[\chi]$ with $\chi \in \mathbb{R}^{n}$, denotes that $p(\chi)$ belongs to the set of polynomials in the variables $\left\{\chi_{1}, \chi_{2}, \cdots, \chi_{n}\right\}$ with coefficients in $\mathbb{R}$. For $x_{1}, x_{2} \in \mathbb{R}^{n},\left(x_{1}, x_{2}\right)$ denotes $\left[x_{1}^{T}, x_{2}^{T}\right]^{T}$. A function $\beta: \mathbb{R}_{\geq 0} \rightarrow \mathbb{R}_{\geq 0}$ is said to be of class $\mathcal{K}$ if it is continuous, zero at zero and strictly increasing. It is said to be of class $\mathcal{K}_{\infty}$ if it is of class $\mathcal{K}$, and it is unbounded. A function $\beta: \mathbb{R}_{\geq 0} \times \mathbb{R}_{\geq 0} \rightarrow \mathbb{R}_{\geq 0}$ is said to be of class $\mathcal{K} \mathcal{L}$ if $\beta(\cdot, t)$ is of class $\mathcal{K}$ for each $t \geq 0$, and $\beta(s, \cdot)$ is non-increasing and satisfies $\lim _{t \rightarrow \infty} \beta(s, t)=0$ for each $s \geq 0$. Recall that a function $f: \mathbb{R} \rightarrow \mathbb{R}^{n}$ is said to be piecewise-continuous on an interval $J \subset \mathbb{R}$ if for every bounded subinterval $J_{0} \subset J, f$ is continuous for all $t \in J_{0}$ except, possibly, at a finite number of points where $f$ may have discontinuities. It is right-continuous at $t$ if $f(t)=\lim _{\theta \uparrow t} f(\theta) \triangleq \lim _{\theta \rightarrow t, \theta>t} f(\theta)$.

\section{Problem formulation}

Consider the affine nonlinear control system given by

$$
\dot{x}=f(x)+g(x) u,
$$


where $f(\cdot), g(\cdot)$ are sufficiently smooth functions on a neighborhood of the origin $\mathrm{x}=0$ denoted by $\mathcal{D}, x \in \mathbb{R}^{n}$ and $u \in \mathbb{R}^{m}$ are the state and the input, respectively. Suppose that there exists a sufficiently smooth function $K(\cdot)$ which defines the continuous-time stabilizer $u=K(x)$. The interconnection between the previous controller and the continuous-time system (1) yields

$$
\dot{x}=f_{n}(x):=f(x)+g(x) K(x) .
$$

H.1 We suppose that system (2) has a well-defined solution $x(t) \in \mathcal{D}$ on the interval $\left[t_{0},+\infty\right)$ for any initial condition $x\left(t_{0}\right)=x_{0} \in \mathcal{D}$.

Note that $\mathcal{D}$ is an invariant set for the closed-loop system (2). We consider the sampled-data implementation of the controller under the following assumptions:

H.2 The control is piecewise constant, calculated based on the sampled-data version of the state

$$
u(t)=K\left(x\left(t_{k}\right)\right), \quad \forall t \in\left[t_{k}, t_{k+1}\right), \quad \forall k \in \mathbb{N} .
$$

H.3 The set of sampling instants $\left\{t_{k}\right\}_{k \in \mathbb{N}}$ satisfies

$$
0<t_{k+1}-t_{k} \leq \bar{h}, \quad \forall k \in \mathbb{N}
$$

for a given MSI $\bar{h}$, and $\lim _{k \rightarrow \infty} t_{k}=+\infty$.

H.4 For any initial condition $x\left(t_{0}\right)=x_{0} \in \mathcal{D}$, the system

$$
\dot{x}(t)=f(x(t))+g(x(t)) K\left(x_{0}\right),
$$

admits a unique solution $x(t)$ originating from $x_{0}$ which is defined on the interval $\left[t_{0}, t_{0}+\bar{h}\right)$ and $x(t) \in \mathcal{D}$.

We obtain the closed-loop sampled-data system (see also Fig. 1):

$$
\begin{aligned}
& \dot{x}(t)=f(x(t))+g(x(t)) K\left(x\left(t_{k}\right)\right), \\
& \forall t \in\left[t_{k}, t_{k+1}\right), \quad \forall k \in \mathbb{N} .
\end{aligned}
$$

If assumptions H.1-H.4 hold, then the system solution $x(t)$ is constructed in an iterative manner by integrating (5) over the interval $\left[t_{k}, t_{k+1}\right)$, and using $x\left(t_{k+1}\right)=$ $\lim _{t \uparrow t_{k+1}} x(t)$ (see [23] for a similar construction). Assumption H.4 can be satisfied if, for example, $f(\cdot)$ and $g(\cdot)$ are globally Lipschitz.

We consider the following notions of stability:

Definition 2.1 The equilibrium point $x=0$ of system (5) is locally uniformly asymptotically stable in a neighborhood $\mathcal{D}_{0}$ of the equilibrium, if there exists a class $\mathcal{K} \mathcal{L}$ function $\beta(\cdot, \cdot)$, such that

$$
|x(t)| \leq \beta\left(\left|x\left(t_{0}\right)\right|, t-t_{0}\right), \quad \forall t \geq t_{0}, \quad \forall x\left(t_{0}\right) \in \mathcal{D}_{0} .
$$

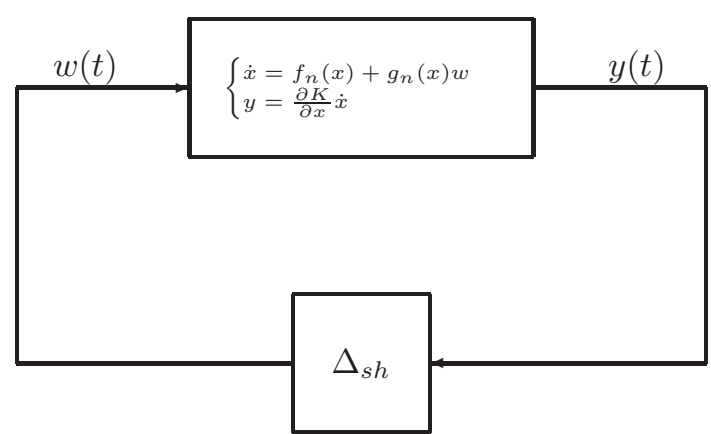

Fig. 2. An equivalent representation of the sampled-data system (5).

In this case $\mathcal{D}_{0}$ is an estimate of the domain of attraction of $x=0$. The equilibrium point $x=0$ is globally uniformly asymptotically stable if (6) is satisfied for any initial state $x\left(t_{0}\right) \in \mathbb{R}^{n}$ (i.e. $\mathcal{D}_{0}=\mathbb{R}^{n}$ ).

Definition 2.2 The equilibrium point $x=0$ of system (5) is locally uniformly exponentially stable in a neighborhood $\mathcal{D}_{0}$ of the equilibrium, if (6) is satisfied with

$$
\beta(s, t)=\varrho s e^{-\lambda t}, \quad \varrho>0, \lambda>0 .
$$

In this case $\mathcal{D}_{0}$ is an estimate of the domain of attraction of $x=0$. The equilibrium point $x=0$ is globally uniformly exponentially stable if this condition is satisfied for any initial state $x\left(t_{0}\right) \in \mathbb{R}^{n}$, (i.e. $\mathcal{D}_{0}=\mathbb{R}^{n}$ ).

The problem under study is formalized as follows.

Problem: Find a criterion for the asymptotic/ exponential stability of the equilibrium point $x=0$ of the sampled-data system (5).

\section{Modeling of sampled-data systems}

The objective of this section is to present an equivalent representation of the sampled-data system (5). This representation has interesting properties from a robust control point of view. The system (5) can be written as

$\dot{x}(t)=f_{n}(x(t))+g_{n}(x(t)) w(t), \quad \forall t \in\left[t_{k}, t_{k+1}\right), \quad k \in$

where $f_{n}(x)=f(x)+g(x) K(x), g_{n}(x)=g(x)$ and $w(t)=K\left(x\left(t_{k}\right)\right)-K(x(t))$. Note that $f_{n}(x)$ represents the dynamics of the nominal, continuous-time, closedloop system, i.e. the dynamics without the sampled-data implementation. $w(t)$ represents the effect of sampling and the variations of the sampling intervals. From (8), the sampled-data system (5) can be represented by the 
equivalent feedback connection of the system

$$
\mathcal{G}:\left\{\begin{array}{l}
\dot{x}=f_{n}(x)+g_{n}(x) w \\
y=\frac{\partial K}{\partial x} \dot{x}
\end{array}\right.
$$

with the reset integrator

$$
\begin{aligned}
& \Delta_{s h}: w(t)=K\left(x\left(t_{k}\right)\right)-K(x(t))=-\int_{t_{k}}^{t} y(\tau) d \tau, \\
& \forall t \in\left[t_{k}, t_{k+1}\right), \forall k \in \mathbb{N} .
\end{aligned}
$$

This representation is shown in Fig. 2. In the next section, we will show how this model will be used for stability analysis of the sampled-data system.

\section{Main results}

In the following, we provide the main results of this paper.

Theorem 1 Consider the sampled-data system (5) under hypotheses H.1-H.4, and the equivalent representation (9), (10). Consider the notations $y(t)$ and $w(t)$ defined in (9) and (10), respectively. Assume that:

I) there exists a continuous function $\mathcal{S}(y, w)$ which satisfies the following integral property

$$
\int_{t_{k}}^{t} \mathcal{S}(y(\theta), w(\theta)) d \theta \leq 0, \quad t \in\left[t_{k}, t_{k+1}\right), \quad \forall k \in \mathbb{N},
$$

along all system solutions $x(t)$ originating in $\mathcal{D}$;

II) there exist a differentiable positive definite function $V: \mathcal{D} \rightarrow \mathbb{R}^{+}$, class $\mathcal{K}$ functions $\beta_{1}$ and $\beta_{2}$ and $\alpha>0$ which satisfy:

$$
\beta_{1}(|x|) \leq V(x) \leq \beta_{2}(|x|), \quad \forall x \in \mathcal{D},
$$

$$
\begin{aligned}
& \dot{V}(x(t))+\alpha V(x(t)) \leq e^{-\alpha \tau(t)} \mathcal{S}(y(t), w(t)), \\
& \forall t \in\left[t_{k}, t_{k+1}\right), \forall x(t) \in \mathcal{D}, \\
& \text { with } \tau(t)=t-t_{k} .
\end{aligned}
$$

Then, the equilibrium $x=0$ of the system (5) is locally uniformly asymptotically stable, and the decay rate of the function $V(x(t))$ is at least $\alpha$. Moreover, the set $\mathcal{L}_{c^{*}}$ defined by the maximal sub-level set of $V$ contained in $\mathcal{D}$

$$
c^{*}=\max _{\mathcal{L}_{c} \subset \mathcal{D}} c
$$

is an estimate of the domain of attraction, where $\mathcal{L}_{c}$ is the sub-level set defined by $V(\cdot)$ and a scalar $c>0$

$$
\mathcal{L}_{c}:=\left\{x \in \mathbb{R}^{n}: V(x) \leq c\right\} .
$$

Finally, if all the conditions are satisfied for $\mathcal{D}=\mathbb{R}^{n}$, with class $\mathcal{K}_{\infty}$ functions $\beta_{1}$ and $\beta_{2}$, then the equilibrium $x=0$ is globally uniformly asymptotically stable.

Proof. First note that $w(t)$ and $y(t)$ are right-continuous, thus the integral term in (11) exists. Consider the following function

$$
\begin{aligned}
& W(t)=V(x(t)) e^{\alpha \tau(t)}-\int_{t_{k}}^{t} \mathcal{S}(y(\theta), w(\theta)) d \theta \\
& \forall t \in\left[t_{k}, t_{k+1}\right), \quad \forall k \in \mathbb{N} .
\end{aligned}
$$

The condition (13) yields

$$
\dot{W}(t) \leq 0, \quad \forall t \in\left[t_{k}, t_{k+1}\right), \quad \forall x(t) \in \mathcal{D} .
$$

The last equation yields

$$
V(x(t)) e^{\alpha \tau(t)}-\int_{t_{k}}^{t} \mathcal{S}(y(\theta), w(\theta)) d \theta \leq V\left(x\left(t_{k}\right)\right) .
$$

From the integral property in (11), it is easy to see that

$$
\begin{aligned}
& V(x(t)) \leq e^{-\alpha \tau(t)} V\left(x\left(t_{k}\right)\right), \\
& \forall t \in\left[t_{k}, t_{k+1}\right), \quad \forall x(t) \in \mathcal{D} .
\end{aligned}
$$

Clearly, the set $\mathcal{L}_{c^{*}}$ is positively invariant [25], and it is the largest sub-level set of $V$ contained in $\mathcal{D}$. Consider an initial condition $x_{0} \in \mathcal{L}_{c^{*}}$. From the continuity of the solution $x(t),(18)$ leads to

$$
\begin{aligned}
& V(x(t)) \leq e^{-\alpha\left(t-t_{0}\right)} V\left(x\left(t_{0}\right)\right) \\
& \forall t \geq t_{0}, \quad \forall x_{0} \in \mathcal{L}_{c^{*}} .
\end{aligned}
$$

From (12) and (19), we see that for any solution with $x\left(t_{0}\right) \in \mathcal{L}_{c^{*}}$

$$
\begin{aligned}
|x(t)| & \leq \beta_{1}^{-1}\left(V\left(x\left(t_{0}\right)\right) e^{-\alpha\left(t-t_{0}\right)}\right) \\
& \leq \beta_{1}^{-1}\left(\beta_{2}\left(\left|x\left(t_{0}\right)\right|\right) e^{-\alpha\left(t-t_{0}\right)}\right) \\
& :=\beta\left(\left|x\left(t_{0}\right)\right|, t-t_{0}\right), \quad \forall t \geq t_{0}, \quad \forall x\left(t_{0}\right) \in \mathcal{L}_{c^{*}}
\end{aligned}
$$

The function $\beta(\cdot, \cdot)$ can be easily seen to be a class $\mathcal{K} \mathcal{L}$ function. This shows that $x=0$ is locally uniformly asymptotically stable. Finally, it is trivial to see that if all the conditions are satisfied globally, with a class $\mathcal{K}_{\infty}$ functions $\beta_{1}$ and $\beta_{2}$, then $x=0$ is globally uniformly asymptotically stable. This completes the proof.

Corollary 4.1 Suppose that all the conditions of Theorem 1 are satisfied with

$$
\begin{aligned}
& \beta_{1}(|x|) \geq k_{1}|x|^{q}, \beta_{2}(|x|) \leq k_{2}|x|^{q}, \\
& \text { for some } k_{1}, k_{2}, q>0 .
\end{aligned}
$$


Then, the equilibrium $x=0$ is locally exponentially stable. Moreover, the sub-level set $\mathcal{L}_{c^{*}}$ defined in (15) and (14), is an estimate of the domain of attraction. If the conditions hold globally, then $x=0$ is globally exponentially stable.

Proof. Following the same steps as in the proof of Theorem 1, we get

$$
V(x(t)) \leq e^{-\alpha\left(t-t_{0}\right)} V\left(x\left(t_{0}\right)\right), \quad \forall t \geq t_{0}, \quad \forall x_{0} \in \mathcal{L}_{c^{*}} .
$$

Thus, from (12) and (20)

$$
\begin{aligned}
|x(t)| & \leq\left(\frac{V\left(x\left(t_{0}\right)\right) e^{-\alpha\left(t-t_{0}\right)}}{k_{1}}\right)^{1 / q} \leq\left(\frac{k_{2}\left|x\left(t_{0}\right)\right|{ }^{q} e^{-\alpha\left(t-t_{0}\right)}}{k_{1}}\right)^{1 / q} \\
& =\left(\frac{k_{2}}{k_{1}}\right)^{1 / q}\left|x\left(t_{0}\right)\right| e^{-(\alpha / q)\left(t-t_{0}\right)}, \forall t \geq t_{0}, \forall x\left(t_{0}\right) \in \mathcal{L}_{c^{*}}
\end{aligned}
$$

This shows that $x=0$ is locally exponentially stable. If the conditions hold globally, global exponential stability is trivial.

Remark 4.1 The result in Theorem 1 is a general, theoretical one. In particular, conditions (11) and (13) are not easily verified, as they require the knowledge of all the trajectories of the system. In the following section, we show how Theorem 1 can be modified in a constructive manner and we provide tractable numeric criteria for the case of polytopic and polynomial systems.

\section{Constructive analysis conditions}

Characterizing candidate functions $\mathcal{S}(\cdot, \cdot)$ in Theorem 1 which satisfy the inequality (11), requires studying the properties of the reset integrator (10). It has been studied from the operator theory point of view, and it has two interesting properties. It has been shown in $[21,31]$ that $\Delta_{s h}$ has a bounded gain. Also, an anti-passivity property was found by Fujioka in [15]. The properties are stated in the following two lemmas which are adapted from [36]. They are based on the work in [15].

Lemma 5.1 (Small gain property) [36] Consider the reset integrator in (10). Then, for any $y \in$ $L_{2}^{m}\left[t_{k}, t_{k+1}\right)$ and $0<X^{T}=X \in \mathbb{R}^{m \times m}$ we have the following inequality:

$$
\int_{t_{k}}^{t}\left(w^{T}(\theta) X w(\theta)-\delta_{0}^{2} y^{T}(\theta) X y(\theta)\right) d \theta \leq 0
$$

for all $t \in\left[t_{k}, t_{k+1}\right)$, where $\delta_{0}=\frac{2}{\pi} \bar{h}$.

Lemma 5.2 (Anti-passivity property) [36] Consider the reset integrator in (10). Then, for any $y \in L_{2}^{m}\left[t_{k}, t_{k+1}\right)$ and $0 \leq Y^{T}=Y \in \mathbb{R}^{m \times m}$, we have the following inequality:

$$
\int_{t_{k}}^{t}\left(w^{T}(\theta) Y y(\theta)+y^{T}(\theta) Y w(\theta)\right) d \theta \leq 0,
$$

for all $t \in\left[t_{k}, t_{k+1}\right)$.

In [15], the properties of $\Delta_{s h}$ have been used in the LTI context, and they lead to LMI stability conditions, which are based on Integral Quadratic Constraints (IQC) [30]. Local stability conditions in the form of LMIs have been proposed for the bilinear case in [36]. The method depends on the properties of $\Delta_{s h}$, it is based on the analysis of invariant sets [3] and dissipativity theory [45]. It is important to notice that the extension of the previous results to a more general class of nonlinear systems is not direct. Next, we show how Theorem 1 along with the properties in Lemma 5.1 and Lemma 5.2, can be used to provide a stability criterion for the case of affine nonlinear systems with sampled-data control.

Corollary 5.1 Consider the sampled-data system (5) under hypotheses H.1-H.3, for the case where $f(\cdot), g(\cdot)$ and $K(\cdot)$ are continuously differentiable functions on $\mathcal{D}$. Suppose that there exist $\alpha>0,0 \leq X^{T}=X \in \mathbb{R}^{m \times m}$, $0 \leq Y^{T}=Y \in \mathbb{R}^{m \times m}$, and a differentiable positive definite function $V: \mathcal{D} \rightarrow \mathbb{R}^{+}$, which satisfies (12), such that

$$
\begin{aligned}
& \frac{\partial V}{\partial x}\left(f_{n}(x)+g_{n}(x) w\right)+\alpha V(x) \leq\left(-\delta_{0}^{2}\left\|\frac{\partial K}{\partial x}\left(f_{n}(x)+g_{n}(x) w\right)\right\|_{X}^{2}\right. \\
& \left.+\|w\|_{X}^{2}+2\left\langle\frac{\partial K}{\partial x}\left(f_{n}(x)+g_{n}(x) w\right), w\right\rangle_{Y}\right) e^{-\alpha \bar{h} l}, \forall l \in\{0,1\}, \quad
\end{aligned}
$$

for all $x \in \mathcal{D}$ and $w \in \mathbb{R}^{m}$. Then the equilibrium $x=0$ of the system (5) is locally uniformly asymptotically stable and the decay rate of the function $V(x(t))$ is $\alpha$. Moreover, the set $\mathcal{L}_{c^{*}}$ defined in (15) and (14), is an estimate of the domain of attraction.

Proof. The proof is based on constructing the solution of the sampled-data system (5) in the same manner as in Theorem 3.3 in [25], and proving the stability of the system such as in Theorem 1.

Given $x_{0} \in \mathcal{L}_{c^{*}}$, the system dynamics over the interval $\left[t_{0}, t_{1}\right)$ are given by

$\dot{x}(t)=\mathcal{F}_{0}(x(t))=f(x(t))+g(x(t)) K\left(x\left(t_{0}\right)\right), \quad \forall t \in\left[t_{0},((2))\right)$

Since $f(\cdot)$ and $g(\cdot)$ are continuously differentiable, then using Theorem 3.2 in [25] we have that $\mathcal{F}_{0}$ is locally Lipschitz continuous on $\mathcal{D}$. Thus, using Theorem 3.1 in [25], we have that $\exists \xi>0$ for which $\dot{x}=\mathcal{F}_{0}(x)$ has a unique solution over $\left[t_{0}, t_{0}+\xi\right]$, with $t_{0}+\xi<t_{1}$. 
Consider $\eta(t):=\frac{e^{-\alpha\left(t-t_{0}\right)}-e^{-\alpha \bar{h}}}{1-e^{-\alpha \bar{h}}} \in[0,1], \forall t \in\left[t_{0}, t_{0}+\xi\right]$. Then, by multiplying $(23)$ by $\eta(t)$ for $l=0$, and by $1-\eta(t)$ for $l=1$ we obtain

$\dot{V}(x(t))+\alpha V(x(t)) \leq e^{-\alpha\left(t-t_{0}\right)} \mathcal{S}(y(t), w(t)), \forall t \in\left[t_{0}, t_{0}+\xi\right]$, Thus,

with

$$
\mathcal{S}(y(t), w(t))=\left[\begin{array}{c}
y(t) \\
w(t)
\end{array}\right]^{T}\left[\begin{array}{rr}
-\delta_{0}^{2} X & Y \\
Y & X
\end{array}\right]\left[\begin{array}{c}
y(t) \\
w(t)
\end{array}\right]
$$

Now, consider the following function
From the uniform continuity of the solution (29) we have that

$$
x\left(t_{f}\right)=x\left(t_{0}\right)+\int_{t_{0}}^{t_{f}} \mathcal{F}_{0}(x(s)) d s .
$$$$
x(t)=x\left(t_{0}\right)+\int_{t_{0}}^{t} \mathcal{F}_{0}(x(s)) d s, \quad t \in\left[t_{0}, t_{f}\right]
$$

is a solution on $\left[t_{0}, t_{f}\right]$. This contradicts the fact that $\left[t_{0}, t_{f}\right)$ is a maximal interval of existence of a solution, and the solution can then be extended to $t_{1}$. Moreover, using the same steps as in (25)-(28).

$W(t)=V(x(t)) e^{\alpha\left(t-t_{0}\right)}-\int_{t_{0}}^{t} \mathcal{S}(y(\theta), w(\theta)) d \theta, \forall t \in\left[t_{0}, t_{0}+\xi\right] . \quad V(x(t)) \leq e^{-\alpha\left(t-t_{0}\right)} V\left(x\left(t_{0}\right)\right), \quad \forall t \in\left[t_{0}, t_{1}\right]$.

The condition (25) yields $\dot{W}(t) \leq 0$ for all $t \in\left[t_{0}, t_{0}+\xi\right]$, which implies steps on the intervals $\left[t_{k}, t_{k+1}\right)$ for all $k \in \mathbb{N}$, and follows the proof of Theorem 1 .

$V(x(t)) e^{\alpha\left(t-t_{0}\right)}-\int_{t_{0}}^{t} \mathcal{S}(y(\theta), w(\theta)) d \theta \leq V\left(x\left(t_{0}\right)\right)$,

Remark 5.1 Note that the hypothesis H.4 which was used in Theorem 1 is no longer needed in Corollary 5.1, where only geometric properties are being used. This is due to the fact that we show the existence of solutions in Corollary 5.1, while in Theorem 1 we needed the existence of solutions in order to prove the stability properties.

Finally, by using Lemma 5.1 and Lemma 5.2 we have that

$$
V(x(t)) \leq e^{-\alpha\left(t-t_{0}\right)} V\left(x\left(t_{0}\right)\right), \quad \forall t \in\left[t_{0}, t_{0}+\xi\right] .
$$

From equation (28) we have that $x\left(t_{0}+\xi\right) \in \mathcal{L}_{c^{*}}$, and thus we can apply again Theorem 3.2 in [25] to extend the solution. Now let us suppose that the solution can not be extended for $\left[t_{0}, t_{1}\right)$. Then, $\exists t_{f}<t_{1}$ such that the solution does not exist for $t>t_{f}$. Let $\left[t_{0}, t_{f}\right)$ be the maximal interval of existence of a solution. The solution of $(24)$ is

$$
x(t)=x\left(t_{0}\right)+\int_{t_{0}}^{t} \mathcal{F}_{0}(x(s)) d s, \quad t \in\left[t_{0}, t_{f}\right) .
$$

Note that from the continuity of $\mathcal{F}_{0}$ and the fact that the solution lies inside the compact set $\mathcal{L}_{c^{*}}$, then $\exists \Lambda>0$ such that

$$
\left|\mathcal{F}_{0}(x(t))\right| \leq \Lambda, \quad \forall t \in\left[t_{0}, t_{f}\right)
$$

and for any $s_{1}, s_{2} \in\left[t_{0}, t_{f}\right)$ we have

$$
\left|x\left(s_{2}\right)-x\left(s_{1}\right)\right|=\left|\int_{s_{1}}^{s_{1}} \mathcal{F}_{0}(x(s)) d s\right| \leq\left(s_{2}-s_{1}\right) \Lambda,
$$

which shows that the solution (29) is uniformly continuous on $\left[t_{0}, t_{f}\right)$. Consider

$$
x\left(t_{f}\right):=x\left(t_{0}\right)+\lim _{t \rightarrow t_{f}} \int_{t_{0}}^{t} \mathcal{F}_{0}(x(s)) d s .
$$

Remark 5.2 From the dissipativity theory point of view, the inequalities in (23) show that the system (9) is exponentially dissipative [18,8], with a storage function $V(x)$, and with supply rate functions $\mathcal{S}(y, w)$ and $e^{-\alpha \bar{h}} \mathcal{S}(y, w)$ respectively, where $\mathcal{S}$ is defined in (26). This can be seen from the Remark 2.8 in [8].

Remark 5.3 The quadratic function in (26) is established using the properties in Lemma 5.1 and Lemma 5.2. Providing new characterizations of $\Delta_{\text {sh }}$ can lead to other candidates of supply rate functions.

In the following, we show how to obtain numerical criteria using Corollary 5.1. We consider the cases of polytopic and polynomial systems.

\subsection{Case study 1: polytopic systems}

Consider the problem formulation in Section 2, when the system (2) has the polytopic representation $[2,40]$

$$
\dot{x}(t)=A(x(t)) x(t)+B(x(t)) u(t),
$$

where $A(\cdot) \in \mathbb{R}^{n \times n}, B(\cdot) \in \mathbb{R}^{n \times m}$ are continuously differentiable functions which satisfy:

$\left.[A(x), B(x)] \in \operatorname{conv}\left\{\left[A_{1}, B_{1}\right],\left[A_{2}, B_{2}\right], \cdots,\left[A_{p}, B_{p}\right]\right\}, \quad \forall x \notin 3 \mathbb{D}\right)$

Here $A_{i}, B_{i}, \forall i \in\{1,2, \cdots, p\}$ are known matrices of appropriate dimensions. Suppose that a continuous-time 
controller $u(t)=F x(t)$ stabilizes the equilibrium locally asymptotically in $\mathcal{D}$, where $F \in \mathbb{R}^{m \times n}$. Consider the sampled-data implementation of the last controller

$$
u(t)=F x\left(t_{k}\right), \quad \forall t \in\left[t_{k}, t_{k+1}\right) .
$$

The following corollary provides criteria for the stability of the sampled-data systems (33) and (35).

Corollary 5.2 Consider the sampled-data system in (33) and (35). Suppose there exist symmetric positive definite matrices $X, Y \in \mathbb{R}^{m \times m}, P \in \mathbb{R}^{n \times n}$, matrices $P_{2}, P_{3} \in \mathbb{R}^{n \times n}$, and a scalar $\alpha>0$ such that the following LMIs are feasible $\lambda_{i}(x)$, and having the sum over the resulting inequalities yield:

$$
\begin{aligned}
& 2 x^{T} P((A(x)+B(x) F) x+B(x) w)+\alpha x^{T} P x \\
\leq & \left(-\delta_{0}^{2}\|F((A(x)+B(x) F) x+B(x) w)\|_{X}^{2}+\|w\|_{X}^{2}\right. \\
& \left.+2\langle F((A(x)+B(x) F) x+B(x) w), w\rangle_{Y}\right) e^{-\alpha \bar{h} l}, \\
& \forall l \in\{0,1\}, \quad \forall x \in \mathcal{D}, \quad \forall w \in \mathbb{R}^{m},
\end{aligned}
$$

where the following fact from the descriptor method [14] has been used:

$$
\left(x^{T} P_{2}^{T}+\dot{x}^{T} P_{3}^{T}\right)(-\dot{x}+A(x) x+B(x) F x+B(x) w)=0 .
$$

The proof follows from Corollary 5.1.

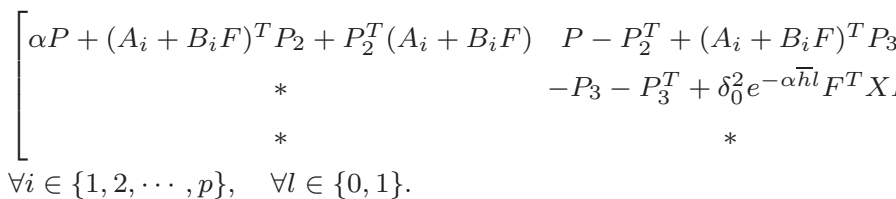

Then the equilibrium $x=0$ of the sampled-data system (33) and (35) is locally asymptotically stable. If for some $c>0$ we define

$$
\mathcal{E}_{c}:=\left\{x \in \mathbb{R}^{n}: x^{T} P x \leq c^{*}\right\}
$$

then an estimate of the domain of attraction is given by

$$
c^{*}=\max _{\mathcal{E}_{c} \subset \mathcal{D}} c .
$$

Proof. Consider a quadratic function $V(x)=x^{T} P x$, vectors $x \in \mathbb{R}^{n}, z \in \mathbb{R}^{n}$ and $w \in \mathbb{R}^{m}$. Multiplying the LMIs (36) by $(x z w)$ from the right, and its transpose from the left implies $\mathcal{E}_{c^{*}}$, where

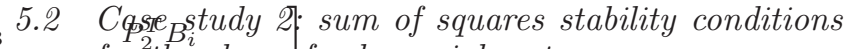

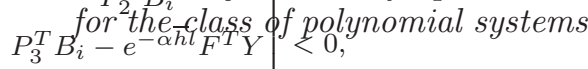

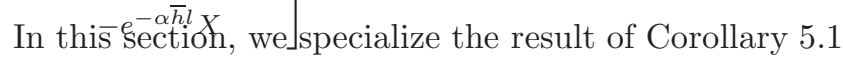
for the class of affine(prolynomial sampled-data systems using SOS decomposition and semi-definite programming techniques $[38,37]$. We formulate a constructive method to find functions $V(\cdot)$ and $\mathcal{S}(\cdot, \cdot)$ which satisfy the proposed stability conditions (23) in Corollary 5.1 for the global stability case.

Consider the stability problem defined in Section 2, for the particular case where $f(\cdot), g(\cdot)$ and $K(\cdot)$ are polynomial functions. System (9) will be defined by polynomial functions $F(x, w):=f_{n}(x)+g_{n}(x) w$ and $G(x, w):=$ $\frac{\partial K}{\partial x} F(x, w)$ :

$$
\left\{\begin{array}{l}
\dot{x}=F(x, w), \\
y=G(x, w) .
\end{array}\right.
$$

When looking for a polynomial function $V(x)$, checking the dissipativity inequalities (23) is a problem of checking the non negativity of polynomials. We will use the SOS technique [38].

Definition 5.1 [37] A multivariate polynomial $p(\chi) \in$ $2 x^{T} P z+\alpha x^{T} P x+2\left(x^{T} P_{2}^{T}+z^{T} P_{3}^{T}\right)\left(-z+A_{i} x+B_{i} F x+\mathbb{R}_{B_{i}} w_{w} j_{s}\right.$ said to be a sum of squares (SOS), if there exist $\leq\left(-\delta_{0}^{2}\|F z\|_{X}^{2}+\|w\|_{X}^{2}+2\langle F z, w\rangle_{Y}\right) e^{-\alpha \bar{h} l}$ $\forall i \in\{1,2, \cdots, p\}, \quad \forall l \in\{0,1\}$.

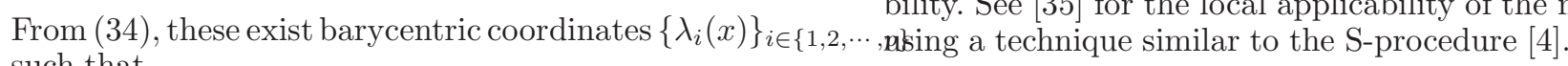
such that

$A(x)=\sum_{i=1}^{p} \lambda_{i}(x) A_{i}, \quad B(x)=\sum_{i=1}^{p} \lambda_{i}(x) B_{i}, \quad x \in \mathcal{D}(39)$

For $z=\dot{x}=(A(x)+B(x) F) x+B(x) w$, and by multiplying each of the inequalities (38) by the appropriate
Corollary 5.3 Consider the sampled-data system (5) in the case where $f(x), g(x)$ and $K(x)$ are polynomial functions, or the equivalent representation (41) and (10). Suppose that there exist a polynomial function $V(x) \in$ $\mathbb{R}[x]$ of degree $2 d, 0<X^{T}=X \in \mathbb{R}^{m \times m}, 0 \leq Y^{T}=Y \in$ $\mathbb{R}^{m \times m}$ and $\alpha>0$ such that the following polynomials are $S O S$ 


\section{$6 \quad$ Illustrative Examples}

$$
\begin{aligned}
\hat{V}(x)= & V(x)-\varphi(x) \\
\rho_{1}(\xi)= & -\frac{\partial V}{\partial x} F(x, w)-\alpha V(x) \\
& +\left[-\left(\frac{2}{\pi} \bar{h}\right)^{2} G^{T}(x, w) X G(x, w)+2 G^{T}(x, w) Y w+w^{T} X w\right]
\end{aligned}
$$$$
\rho_{2}(\xi)=-\frac{\partial V}{\partial x} F(x, w)-\alpha V(x)
$$$$
+\left[-\left(\frac{2}{\pi} \bar{h}\right)^{2} G^{T}(x, w) X G(x, w)+2 G^{T}(x, w) Y w+w^{T} X w\right]
$$

with $\xi=(x, w)$ and $\varphi(x)$ a positive definite polynomial defined by:

$\varphi(x)=\sum_{i=1}^{n} \sum_{j=1}^{d} \epsilon_{i j} x_{i}^{2 j}$, such that $\sum_{j=1}^{d} \epsilon_{i j}>\gamma$ $\forall i=1, \ldots, n$

with $\gamma$ a positive number, and $\epsilon_{i j} \geq 0$ for all $i$ and $j$.

Then, the equilibrium $x=0$ of the sampled-data system is globally uniformly asymptotically stable.

Proof. First, note that from (42) and Proposition 5 from [37], the function $V(x)$ is ensured to be definite positive. Also, it is easily seen that it is radially unbounded $(V(x) \rightarrow \infty$ when $x \rightarrow \infty)$. Therefore, using Lemma 4.3 from [25], there exist class $\mathcal{K}_{\infty}$ functions $\beta_{1}$ and $\beta_{2}$, such that

$$
\beta_{1}(|x|) \leq V(x) \leq \beta_{2}(|x|), \quad \forall x \in \mathbb{R}^{n}
$$

Finally, using the notations $F(x, w):=f_{n}(x)+g_{n}(x) w$ and $G(x, w):=\frac{\partial K}{\partial x} F(x, w)$ in (41), the inequalities (43), (44) implies (23). Thus all the global stability conditions of Corollary 5.1 are satisfied.

Remark 5.4 (Numerical issues) Note that for a fixed $\bar{h}$ and $\alpha$, the sufficient stability conditions in Corollary 5.2 and Corollary 5.3 are numerically tractable. For the polytopic case, checking the conditions of Corollary 5.2 is an LMIs feasibility problem. However, we have restricted our choice to quadratic functions $V(x)=x^{T} P x$ with $P>0$, which might be a source of conservatism. For the polynomial systems case, semidefinite programming and SOS decomposition [39] are used to ensure the applicability of the proposed criterion. In this case, one must also fix $\gamma$ and the degree of $V(\cdot)$. In fact, the choice of the degree of the storage function $V(\cdot)$ is a difficult question [27]. For similar problems, try-and-error methods are being used in the literature [41,7]. This is done by considering small values. Next, these values are increased if the conditions are not satisfied. For both the polytopic and polynomial cases, a line search permits to find an estimate of the value of $\bar{h}$ which ensures stability.
In this section, we will apply the proposed methods on numeric examples. First, we consider a nonlinear system of the form (33) and we apply Corollary 5.2 in order to analyze the stability of the sampled-data system. Then, we consider a benchmark example from the literature. The considered system is polynomial. We apply Corollary 5.3 and show the effectiveness of the proposed $e^{\text {method. }}$

\subsection{Example 1}

Consider the following system

$$
\left(\begin{array}{c}
\dot{x}_{1} \\
\dot{x}_{2}
\end{array}\right)=\left(\begin{array}{cc}
1+0.1 x_{2} & 1 \\
0 & 0.1 \sin \left(x_{1}\right)
\end{array}\right)\left(\begin{array}{l}
x_{1} \\
x_{2}
\end{array}\right)+\left(\begin{array}{l}
0 \\
1
\end{array}\right) u
$$

with the state feedback controller given by the gain $F=\left[\begin{array}{ll}-6 & -4\end{array}\right]$. This controller stabilizes the system in continuous-time. The system will have a polytopic representation given by (39) inside the domain $\mathcal{D}=\{x \in$ $\left.\mathbb{R}^{2}: x_{2} \in[-1,+1]\right\}$. Using Corollary 5.2 we find that the system is stable under aperiodic sampled-data version of the control, for $\bar{h}=0.389$ and $\alpha=0.01$. An estimate of the domain of attraction along with simulations of the system evolution (from various initial conditions with arbitrary sampling intervals over bounded by $\bar{h}$ ) are presented in Fig 3. Note that the estimate of the domain of attraction was not found by performing ad-hoc simulations, but by Corollary 5.2. More precisely, the estimate of the domain of attraction given by $\mathcal{E}_{c^{*}}$ in equation (37), is found numerically by solving an LMI optimization problem. It represents the maximal sub-level set of $V(\cdot)$, contained in the domain $\left\{x \in \mathbb{R}^{2}:\left|x_{2}\right| \leq 1\right\}$. The function $V(\cdot)$ is found from $(36)$, while $c^{*}$ is found by solving an additional LMI problem:

$$
\begin{gathered}
\kappa^{*}=\underset{\min }{\text { s.t. }}\left[\begin{array}{ll}
\kappa & a^{T} \\
a & P
\end{array}\right] \geq 0
\end{gathered}
$$

with $a=\left[\begin{array}{ll}0 & 1\end{array}\right]^{T}$ and $c^{*}=1 / \kappa^{*}$.

\subsection{Example 2}

Consider the following system from [34]

$$
\dot{x}=d x^{2}-x^{3}+u,
$$

with a bounded time-varying $|d| \leq 1$ and a stabilizing control $u=K(x)=-2 x$. Emulating this controller results in a sampled-data system that can be represented 


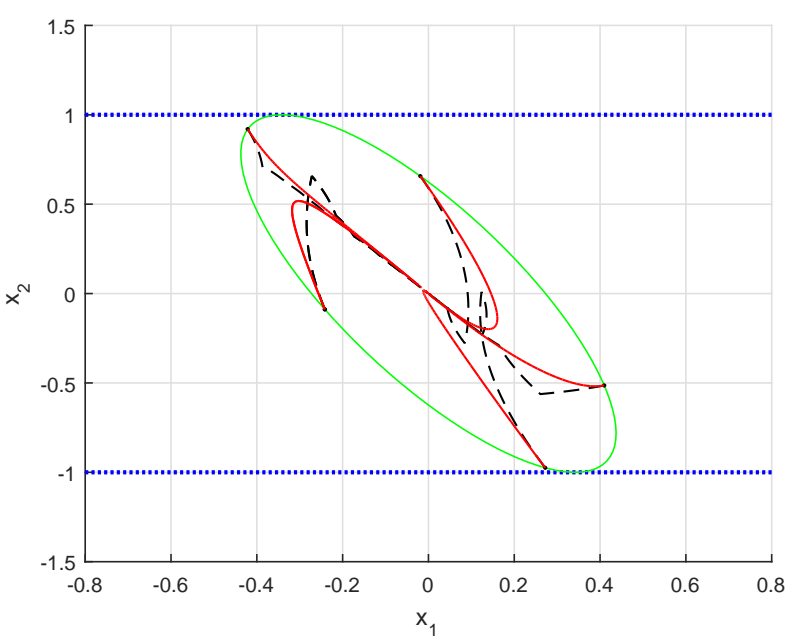

Fig. 3. An estimate of the domain of attraction (in green) for the sampled-data control of the system in Example 6.1, with $\bar{h}=0.389$. Simulations of the continuous (in red) and sampled-data (in dashed black) control of the system, for few initial conditions inside the domain on attraction.

by the reset integrator in (10) and a system (9) described by

$$
\left\{\begin{array}{l}
\dot{x}=d x^{2}-x^{3}-2 x+w, \\
y=-2\left(d x^{2}-x^{3}-2 x+w\right) .
\end{array}\right.
$$

We apply Corollary 5.3 in order to find a storage function of the form $V(x)=a x^{2}+b x^{4}$, such that (42), (43) and (44) are SOS. We choose $\varphi(x)=10^{-3} x^{2}, \alpha=0.1$ and $\bar{h}=0.72$. We intend to test the global stability. In this case, the polynomials (43) and (44) are
The conditions proposed in this paper are found feasible for $\bar{h}=0.72$. The stability conditions have been used in order to find estimates of $\bar{h}$ for several values of the decay rate. The relation between the decay rate $\alpha$ and $\bar{h}$ is illustrated in Table 1 . Note that one cannot expect that the upper bound provided by our method would always be better than the ones provided by the methods in $[34,23]$. Those flexible methods are based on different approaches and can handle a large class of nonlinear systems.

\section{Conclusion}

In this note we have provided sufficient conditions for the stability of nonlinear sampled-data systems, which are affine in the control. The main idea is to use the dissipativity theory to provide an estimate of the MSI. For some particular cases, the proposed methodology leads to numerically tractable conditions. More precisely, the stability criterion for polytopic sampled-data systems is expressed as an LMI feasibility problem, while the case of polynomial sampled-data systems leads to the use of SOS decomposition and semi-definite programming. The method is applied on two examples and it shows that it can provide a good estimate of the MSI. The novelty of this contribution is that it provides a quantitative estimate of the MSI using robust control tools based on the dissipativity theory. It must be noticed that it is possible to enhance the results numerically by studying the reset integrator $\Delta_{s h}$. In fact, providing new characterizations $[16,22]$, can lead to other candidates of supply rate functions, which can be used to decrease the conservatism of the proposed method.

$$
\begin{aligned}
\rho_{1}(\xi)= & -\left(2 a x+4 b x^{3}\right)\left(d x^{2}-x^{3}-2 x+w\right)-\alpha\left(a x^{2}+a x^{4}\right) \\
& +\left[-4 \delta_{0}^{2} X\left(d x^{2}-x^{3}-2 x+w\right)^{2}-4 Y\left(d x^{2}-x^{3}-2 x+w\right)\{\underline{\text { Re }}]\right.
\end{aligned}
$$

$\rho_{2}(\xi)=-\left(2 a x+4 b x^{3}\right)\left(d x^{2}-x^{3}-2 x+w\right)-\alpha\left(a x^{2}+a x^{4}\right)$

$$
+\left[-4 \delta_{0}^{2} X\left(d x^{2}-x^{3}-2 x+w\right)^{2}-4 Y\left(d x^{2}-x^{3}-2 x\right.\right.
$$

where $a, b, X, Y$ are decision variables. Note that the time-varying terms $d$ and $d^{2}$ appear in the polynomial expressions. However, if both (46) and (47) are ensured to be SOS for all the values of $\left(d, d^{2}\right) \in\{(1,0),(1,1),(-1,0),(-1,1)\}$, then they will be SOS for any time-varying $|d| \leq 1$. This is found to be satisfied using the SOSTOOLS [39], for $V(x)=0.77402 x^{2}+0.19911 x^{4}, X=0.47522$ and $Y=0.6230210^{-3}$. By Corollary 5.3, we obtain the global uniform asymptotic stability of the equilibrium $x=0$ of the sampled-data system. This result cannot be obtained when trying a quadratic storage function. Previous works considered this example in the literature for estimating the MSI. In [34], a bound of $\bar{h}=0.368$ is found. In [23], the proposed upper bound is $\bar{h}=0.1428$.

\section{References}

+NXIW. ${ }^{2}$ Bauer, P.J.H. Maas, and W.P.M.H. Heemels. Stability analysis of networked control systems: A sum of squares approach. Automatica, 48(8):1514 - 1524, 2012.

[2] J. Bernussoun, P.L.D. Peres, and J.C. Geromel. A linear $w+$ probotalaming oriented procedure for quadratic stabilization of uncertain systems. Systems \& Control Letters, 13(1):65 72, 1989 .

[3] F. Blanchini. Set invariance in control. Automatica, 35(11):1747 - 1767, 1999.

[4] S. Boyd, L. El Ghaoui, E. Feron, and V. Balakrishnan. Linear Matrix Inequalities in System and Control Theory. Studies in Applied Mathematics. SIAM, 1994.

[5] C. Briat and A. Seuret. A looped-functional approach for robust stability analysis of linear impulsive systems. Systems E Control Letters, 61(10):980 - 988, 2012.

[6] L. Burlion, T. Ahmed-Ali, and F. Lamnabhi-Lagarrigue. On the stability of a class of nonlinear hybrid systems. Nonlinear Analysis: Theory, Methods 83 Applications, 65(12):2236 2247, 2006 .

[7] F. Allgwer C. Ebenbauer. Analysis and design of polynomial control systems using dissipation inequalities and sum of squares. Computers and Chemical Engineering, 30(1012):1590 - 1602, 2006. 
Table 1

The estimates of $\bar{h}$ for different values of the decay rate $\alpha$.

\begin{tabular}{c|c|c|c|c|c|c|c|c|c|c|}
\hline \hline$\alpha$ & 0.1 & 0.2 & 0.3 & 0.4 & 0.5 & 0.6 & 0.7 & 0.8 & 0.9 & 1 \\
\hline $\bar{h}$ & 0.72 & 0.71 & 0.69 & 0.68 & 0.67 & 0.66 & 0.64 & 0.62 & 0.61 & 0.6 \\
\hline
\end{tabular}

[8] V. Chellaboina and W.M. Haddad. Exponentially dissipative nonlinear dynamical systems a nonlinear extension of strict positive realness. Mathematical Problems in Engineering, 1:25-45, 2003.

[9] C. Fiter, L. Hetel, W. Perruquetti, and J.-P. Richard. A state dependent sampling for linear state feedback. Automatica, 48(8):1860 - 1867, 2012.

[10] C. Fiter, H. Omran, L. Hetel, and J.-P. Richard. Tutorial on arbitrary and state-dependent sampling. In European Control Conference (ECC), pages 1440-1445, 2014.

[11] F. Forni, S. Galeani, D. Nešić, and L. Zaccarian. Eventtriggered transmission for linear control over communication channels. Automatica, 50(2):490 - 498, 2013.

[12] E. Fridman. A refined input delay approach to sampled-data control. Automatica, 46(2):421 - 427, 2010.

[13] E. Fridman. Introduction to time-delay and sampled-data systems. In European Control Conference (ECC), pages 1428-1433, 2014.

[14] E. Fridman, A. Seuret, and J.-P. Richard. Robust sampleddata stabilization of linear systems: an input delay approach. Automatica, 40(8):1441 - 1446, 2004.

[15] H. Fujioka. Stability analysis of systems with aperiodic sample-and-hold devices. Automatica, 45(3):771 - 775, 2009.

[16] H. Fujioka. Stability verification of non-uniformly sampleddata systems with a new IQC in continuous-time domain. In International Conference on Control, Automation and Systems, 2011.

[17] R. Goebel, R.G. Sanfelice, and A.R. Teel. Hybrid dynamical systems. IEEE Control Systems, 29(2):28 - 93, 2009.

[18] W.M. Haddad and T. Sadikhov. Dissipative differential inclusions, set-valued energy storage and supply rate maps, and stability of discontinuous feedback systems. Nonlinear Analysis: Hybrid Systems, 8(0):83 - 108, 2013.

[19] L. Hetel, A. Kruszewski, W. Perruquetti, and J. P Richard. Discrete and intersample analysis of systems with aperiodic sampling. IEEE Transactions on Automatic Control, 56(7):696 - 1701, 2011.

[20] P. Hsu and S. Sastry. The effect of discretized feedback in a closed loop system. In 26th IEEE Conference on Decision and Control, pages 1518-1523, 1987.

[21] C.-Y. Kao and B. Lincoln. Simple stability criteria for systems with time-varying delays. Automatica, 40(8):1429 1434, 2004

[22] C.-Y. Kao and D.-R. Wu. On robust stability of aperiodic sampled-data systems - an integral quadratic constraint approach. In American Control Conference (ACC), pages 4871-4876, 2014.

[23] I. Karafyllis and C. Kravaris. Global stability results for systems under sampled-data control. International Journal of Robust and Nonlinear Control, 19:1105 - 1128, 2009.

[24] I. Karafyllis and M. Krstic. Nonlinear stabilization under sampled and delayed measurements, and with inputs subject to delay and zero-order hold. IEEE Transactions on Automatic Control,, 57(5):1141 - 1154, 2012.

[25] H. Khalil. Nonlinear Systems. Prentice-Hall, Upper Saddle River, 2002.
[26] D. Laila, D. Nešić, and A. Astolfi. Sampled-data control of nonlinear systems. In Antonio Loría, Françoise LamnabhiLagarrigue, and Elena Panteley, editors, Advanced Topics in Control Systems Theory, volume 328 of Lecture Notes in Control and Information Science, pages 91-137. Springer London, 2006.

[27] A.Papachristodoulou M. Peet. A converse sum of squares lyapunov result with a degree bound. IEEE Transactions on Automatic Control, 57(9):2281-2293, 2012.

[28] F. Mazenc, M. Malisoff, and T.N. Dinh. Robustness of nonlinear systems with respect to delay and sampling of the controls. Automatica, 49(6):1925 - 1931, 2013.

[29] F. Mazenc and D. Normand-Cyrot. Stabilization of linear input delayed dynamics under sampling. In IEEE 51st Annual Conference on Decision and Control (CDC), 2012.

[30] A. Megretski and A. Rantzer. System analysis via integral quadratic constraints. IEEE Transactions on Automatic Control, 42(6):819 - 830, 1997.

[31] L. Mirkin. Some remarks on the use of time-varying delay to model sample-and-hold circuits. IEEE Transactions on Automatic Control, 52(6):1109 - 1112, 2007.

[32] S. Monaco and D. Normand-Cyrot. Advanced tools for nonlinear sampled-data systems analysis and control. European Journal of Control, 13(23):221 - 241, 2007.

[33] P. Naghshtabrizi, J.P. Hespanha, and A.R. Teel. Exponential stability of impulsive systems with application to uncertain sampled-data systems. Systems $\&$ Control Letters, 57(5):378 $-385,2008$.

[34] D. Nešić, A.R. Teel, and D. Carnevale. Explicit computation of the sampling period in emulation of controllers for nonlinear sampled-data systems. IEEE Transactions on Automatic Control, 54(3):619 - 624, 2009.

[35] H. Omran, L. Hetel, J.-P. Richard, and F. LamnabhiLagarrigue. On the stability of input-affine nonlinear systems with sampled-data control. In European Control Conference $(E C C)$, pages $2585-2590,2013$.

[36] H. Omran, L. Hetel, J.-P. Richard, and F. LamnabhiLagarrigue. Stability analysis of bilinear systems under aperiodic sampled-data control. Automatica, 50(4), 2014.

[37] A. Papachristodoulou and S. Prajna. A tutorial on sum of squares techniques for systems analysis. In American Control Conference, volume 4, pages 2686 - 2700, 2005.

[38] P. Parrilo. Structured semidefinite programs and semialgebraic geometry methods in robustness and optimization. PhD thesis, California Institute of Technology, Pasadena, CA, 2000.

[39] S. Prajna, A. Papachristodoulou, and P.A. Parrilo. Introducing SOSTOOLS: a general purpose sum of squares programming solver. In Proceedings of the 41st IEEE Conference on Decision and Control, volume 1, pages $741-$ 746, 2002.

[40] W.J. Rugh and J.S. Shamma. Research on gain scheduling. Automatica, 36(10):1401 - 1425, 2000.

[41] A. Rantzer S. Prajna, P. Parrilo. Nonlinear control synthesis by convex optimization. IEEE Transactions on Automatic Control, 49(2):310-314, 2004. 
[42] A. Seuret. A novel stability analysis of linear systems under asynchronous samplings. Automatica, 48(1):177 - 182, 2012.

[43] A. Seuret and F. Gouaisbaut. Wirtinger-based integral inequality: Application to time-delay systems. Automatica, 49(9):2860 - 2866, 2013.

[44] J. Willems. Dissipative dynamical systems. part I: general theory. Archive for Rational Mechanics and Analysis, 45(5):321 - 351, 1972.

[45] J. Willems. Dissipative dynamical systems. European Journaol of Control, 13:134 - 151, 2007. 